\title{
Xanthogranulomatous Pyelonephritis: A Narrative Review with Current Perspectives on Diagnostic Imaging and Management, Including Interventional Radiology Techniques
}

\author{
Mark Paul Bolger (D) \\ Jennifer Hennebry \\ Caoimhe Byrne \\ Laura Greene \\ Andreea Stroiescu \\ Joan Heneghan \\ Anthony Gerard Ryan
}

Division of Interventional Radiology, Department of Radiology, University Hospital Waterford, Waterford City, X91 DWX0, Ireland
Correspondence: Anthony Gerard Ryan Division of Interventional Radiology, Department of Radiology, University Hospital Waterford, Ardkeen, Waterford City, X9I DWX0, Ireland

Tel +3535 I 848000

Fax +3535I84857I

Email anthonyryanir@gmail.com

\begin{abstract}
Xanthogranulomatous Pyelonephritis (XGP) is a rare, chronic granulomatous inflammatory condition thought to arise secondary to a combination of obstruction, recurrent bacterial infection and an incomplete immune response resulting in focal or diffuse renal destruction. This destruction may be profound with the potential to infiltrate surrounding tissues and viscera. The imaging features of XGP can be ambiguous, mimicking malignancy, tuberculosis (TB) and malakoplakia earning the title of "the great imitator". Computed tomography (CT) is the mainstay of XGP diagnosis and staging, accurately quantifying the stone burden and staging the renal destruction, including the extent of extra-renal spread. Although some cases in children have been successfully treated with antibiotics alone, nephrectomy remains the most common treatment for XGP in adults. The specific management strategy needs to be tailored to individual patients given the potential constellation of renal and extrarenal abnormalities. Although XGP has classically required open nephrectomy, laparoscopic nephrectomy has an increasing role to play arising from the advancement in laparoscopic skills, technique and instruments. Nephron-sparing partial nephrectomy may be considered in the focal form. Interventional radiology techniques most often play a supportive role, eg, in the initial drainage of associated abscesses, but have rarely achieved renal salvage. This narrative review seeks to synthesise the existing literature and summarise the radiological approach and interventional radiology management situated in a clinical context.
\end{abstract}

Keywords: xanthogranulomatous pyelonephritis, XGP, staghorn calculus

\section{Introduction}

Xanthogranulomatous Pyelonephritis (XGP) is a rare, proliferative chronic granulomatous inflammatory condition characterised by gross renal parenchymal destruction and replacement by focal accumulation of Xanthomatous aggregates of lipid-laden epithelioid macrophages (foam cells). A type of xanthogranulomatous pyelonephritis was first described by Schlagenhaufer in $1916 ;{ }^{1}$ however, the current description was not applied until Osterlind in $1944 .^{2}$ This chronic granulomatous process can result in significant destruction of renal parenchyma and propagation of inflammation into adjacent pararenal tissues and viscera. This narrative review seeks to synthesise the existing literature and summarise the radiological approach and interventional radiology management situated in a clinical context. 


\section{Pathophysiology}

The precise pathophysiology remains incompletely understood, but, given the observed associations, the combination of obstruction and infection are presumed the primary initiators, resulting in an interstitial pyelonephritis, followed by a subsequent chronic granulomatous immune response which fails to completely eradicate the inciting agent. $^{3}$

It seems likely that a common obstructive pathway results in superinfection of static urine with consequent stone formation. Infected debris (usually with relatively indolent agents, please see below), incites the chronic granulomatous inflammatory response with incomplete clearance of the provoking factor(s). Evidence to support the hypothesis that granuloma formation is induced primarily by bacteria includes the presence of bacteria within the granulomas in both intra-and extracellular locations, including within cytoplasmic vacuoles. The failure to completely degrade the bacterial products provokes a chronic inflammatory response, but also suggests a limited/incomplete host immune response. ${ }^{4,5}$.

An increased incidence of calyceal stones and staghorn calculi, recurrent urinary tract infections and chronic interstitial nephritis is well established and these are the most common associations. Similarly, an increased incidence of conditions leading to obstruction such as pyeloureteric junction obstruction, ureteropelvic duplication, ureteral schistosomiasis ${ }^{6}$ and obstructing tumours (including renal and transitional cell carcinomas) has been documented. A case described in a 21-day-old neonate arose secondary to grade $\mathrm{V}$ vesicoureteric reflux. ${ }^{7}$

Despite the presence of a staghorn calculus in the renal pelvis of the majority of affected patients, renal parenchymal destruction and subsequent renal impairment occur secondary to the chronic inflammation and macrophage infiltration as opposed to obstructive uropathy, as the urine can usually bypass the staghorn, which results in a partial, rather than complete obstruction.

The process is usually unilateral and disseminated (involving the entirety of the kidney); however, a more atypical distribution of segmental/focal XGP can also occur in $17 \%$ of cases. ${ }^{8,9}$ The process can extend beyond the kidney into the perinephric and para-renal spaces and in severe, advanced cases may infiltrate adjacent tissues and viscera. The degree of extra-renal extension can be profound, with reported cases of pancreatic, splenic and hepatic infiltration with abscess formation, cutaneous, colonic and duodenal fistulae and rib osteomyelitis also described. ${ }^{10-15}$ Very rare instances of renal amyloidosis secondary to XGP have been reported. ${ }^{16-18 .}$

\section{Epidemiology}

Although XGP occurs most commonly in middle age, mean age at presentation ranging from 45 to 55.2 years, it has been described in neonates and the elderly (described age range from 21 days to 84 years). ${ }^{19-21}$ In adults, the condition affects females twice as often as males and the right kidney more often than left. ${ }^{22}$ No gender or side predominance has been identified in children. $^{23,24}$. In addition to those described above, other predisposing conditions include pelviureteric junction obstruction, ureteropelvic duplication, chronic interstitial nephritis and bladder tumours. ${ }^{8}$ Associations with diabetes mellitus, rheumatoid arthritis, and the metabolic syndrome have also been noted. ${ }^{9,19}$ XGP in the setting of a kidney with chronic pyelonephritis has a reported incidence of $4 \%{ }^{9}$

\section{Clinical Presentation}

Although the vast majority of patients are symptomatic, their presentation is often non-specific with general malaise. Symptoms have usually been present for some time, longer than 6 months in $42 \% .{ }^{22}$ Commonly reported symptoms include flank or abdominal pain, lower urinary tract symptoms, fever, palpable tender mass, gross haematuria and weight loss; however, urinary tract symptoms may be surprisingly minimal, leading to initial misdiagnosis. Patients generally report more than one symptom. ${ }^{19}$ They will often appear to be in poor general condition, some cachectic, suggesting a patient with malignancy or chronic debilitating infection such as Tuberculosis.

\section{Diagnosis \\ Laboratory Findings}

Common laboratory findings at presentation include leucocytosis in $41 \%$ of patients and anaemia in $63 \%$ of patients. ${ }^{25}$ In Korkes' series, the mean initial white cell count was 12.3 x $10^{6} / \mathrm{mL}$ (range 3.5 to 22.5 ) and mean initial haemoglobin was $10.4 \mathrm{~g} / \mathrm{dL}$ (range 7.5 to 14.2). Elevated ESR and CRP are expected. Disordered liver function tests have been observed in a minority of patients which normalise following definitive treatment. ${ }^{26,27}$. Serial urine cytology with demonstration of urinary foam cells has been used to 
confirm the preoperative diagnosis of XGP in a small number of cases. ${ }^{28,29}$

\section{Microbiology}

The two most commonly cultured organisms are Escherichia coli and Proteus mirabilis (35.3\% and 17.6\% respectively), infection with Proteus mirabilis exacerbating the resultant pyelonephritis secondary to urease excretion. $^{25}$ Other causative organisms include Pseudomonas aeruginosa, Klebsiella pneumoniae, Serratia marcescens and Staphylococcus aureus. ${ }^{26,30 .}$ Although cultures were previously thought to be positive in up to $70 \%$ of cases ${ }^{22}$ this has not been replicated in all series, one study of 39 patients demonstrating that over half $(55 \%)$ had sterile urine; ${ }^{9}$ thus XGP cannot be excluded in the setting of sterile urine. If urine cultures are negative, blood cultures, biopsied renal tissue or retrieved calculi may be positive. ${ }^{31,32}$.

A recently published case series of 27 patients with XGP showed 13 patients (48.1\%) had positive urine cultures, the isolated pathogens including Proteus Mirabilis (18.5\%), Escherichia Coli (14.8\%) and Pseudomonas Aeruginosa (7.4\%), while 2 patients had mixed growth (Escherichia coli + Streptococcus constellatus - 3.7\%, Proteus mirabilis + Serratia marcescens $-3.7 \%$ ).

The overall antibiotic resistance profile was also explored in this case series which showed resistance to Quinolones (14.3\%), Amoxicillin/Clavulanic Acid (12.5\%), Piperacillin/Tazobactam (14.3\%), Cegtriaxone (16.6\%), Fosfomycin (33.3\%) and Trimethoprim/ Sulfamethoxazole $(33.3 \%))^{33}$

\section{Stone Composition}

Biochemical analysis of retrieved calculi demonstrates predominantly phosphate stones (typical of struvite, or infection-related calculi) with lesser proportions of Carbapatite, Protein and Whewelite (Calcium oxalate monohydrate).

\section{Gross Pathology}

The typical gross pathological appearance is that of an enlarged kidney with capsular thickening and replacement of parenchyma with yellow, possibly necrotic nodules and a dilated pelvicalyceal system containing stones/debris and variable volumes of pus.

\section{Microscopy}

Histology is characterised by a chronic interstitial pyelonephritis with periglomerular fibrosis. Tubular atrophy and dilatation may be present, with or without thyroidisation. Variable interstitial populations of lymphocytes, plasma cells, neutrophils, multinucleated histiocytic giant cells are observed in addition to the invariable heavy foam cell infiltrate. ${ }^{30,34}$

\section{Diagnostic Imaging}

XGP has gained the title of the "great imitator" because of the overlap of imaging features with other conditions including renal cell carcinoma (RCC), urothelial tumours (TCC), Tuberculosis (TB) and Malakoplakia. This overlap contributes to the frequently observed delay in reaching a specific diagnosis.

There are multiple case reports in the literature of concurrent XGP and synchronous renal malignancy within focal or diffuse XGP which contributes further to this diagnostic confusion. A clear pathophysiologic association between these entities has not been established, one hypothesis being initial tumoural obstruction of the renal collecting system resulting in XGP; however a clear sequence of events in these concurrent cases remains unclear. $^{35,36}$ This possibility of concurrent malignancy requires careful consideration in each case before ascribing all the findings a non-malignant aetiology, especially if nephrectomy is not planned.

\section{Radiographic Techniques}

Conventional radiographs of the abdomen will identify radiopaque staghorn calculi (when present) projected through the expected position of the renal pelvis (Figure 1A); however, not all patients with XGP have a renal calculus, nor do all patients with staghorn calculi have XGP. Other, more subtle, radiographic features include an enlarged renal outline and obscuration of the ipsilateral psoas margin in advanced disease. ${ }^{3}$

Intravenous pyelography is now rarely performed; however pyelographic images following intravenous injection of contrast can still be contributory (Figure 1B) in demonstrating lack of excretion in affected poles. Secondary complications such as fistulae and abscesses can be demonstrated elegantly by fluoroscopy following contrast injection during interventional procedures (Figure 2A and B).

\section{Ultrasound}

Ultrasound will show an enlarged kidney with gross distortion of the normal renal architecture. Staghorn calculi will be seen as large amorphous echogenicities with 

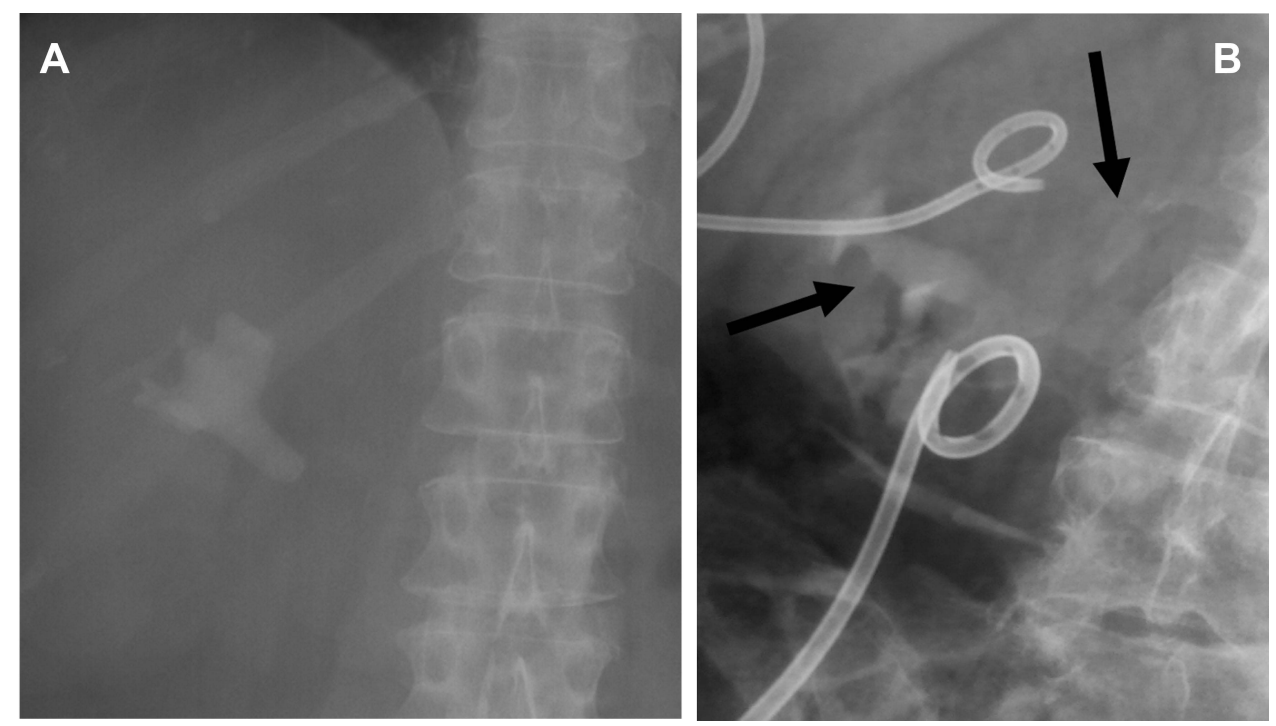

Figure I (A) Large staghorn calculus occupying the right renal pelvis with extension into the calyces in a case of confirmed XGP. (B) IVP in a different patient showing absent excretion in the affected upper pole and normal excretion in the unaffected lower pole (more horizontal arrow). A large irregular calculus is also evident immediately caudal to the right transverse process of L3 (more vertical arrow). The larger drain further caudally is in a psoas abscess and was inserted from the groin.
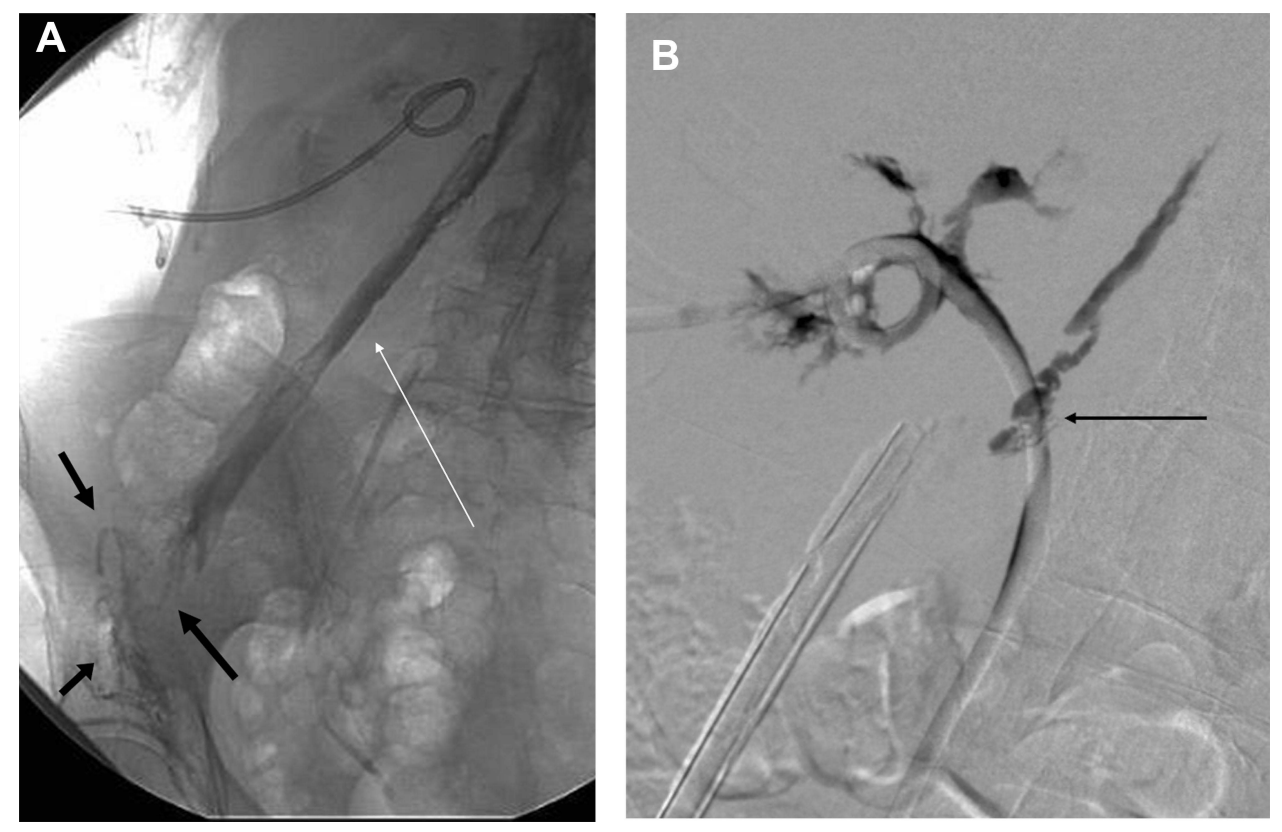

Figure 2 (A) Fluoroscopic image following contrast injection via a nephrostomy catheter demonstrating opacification of a psoas abscess cavity (white arrow) via a sinus from the pyeloureteric junction and opacification of multiple abscess-cutaneous sinuses in the groin (black arrows). (B) Digital subtraction pyelography during contrast injection of a nephro-ureteral stent in the same patient demonstrating opacification of a psoas abscess cavity via a sinus (black arrow) at the level of the pyeloureteric junction. Markedly scarred, ragged calyces and a severely contracted renal pelvis are evident in the affected upper pole moiety.

posterior acoustic shadowing in the renal pelvis. Multiple anechoic or hypoechoic masses may be seen similar to the "bear paw sign" seen on CT, representing the xanthomatous conglomerates (Figure 3A and B). Dilated and multiloculated calyces may also be visualised with internal echoes denoting pyelitis. In focal/segmental XGP, a focal mass may be seen which can be difficult to differentiate from a renal neoplasm on ultrasound alone. Extrarenal extension and abscess formation may also be well demonstrated with ultrasound (Figure 3A and B).

\section{Computed Tomography (CT)}

$\mathrm{CT}$ is the mainstay of the diagnostic imaging assessment of XGP, demonstrating the dilated calyces, changes in 

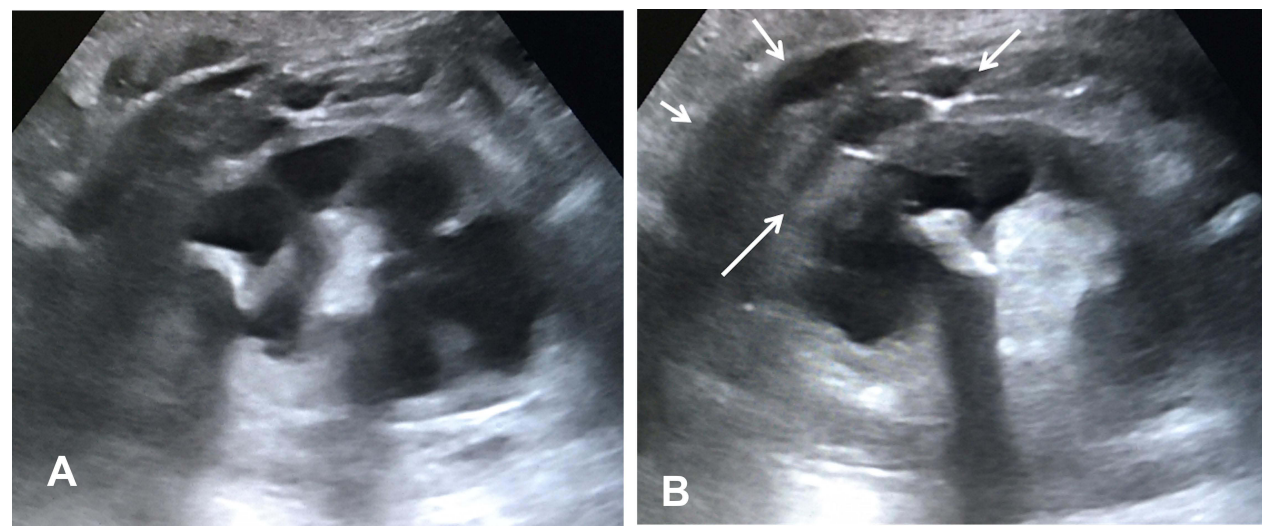

Figure 3 (A) Ultrasound of the kidney showing an "ultrasound bear-paw" with a central calculus and infiltrated fat and dilated, infiltrated calyces peripherally. The overlying cortex is chronically thinned. (B) Further ultrasound image in the same patient showing more marked calculous shadowing and a multilocular extrarenal collection (arrows).

renal size and shape as well as accurately identifying and quantifying the stone burden and associated complications. $^{9}$

The bear-paw sign is a characteristic, moderately specific but not pathognomonic, imaging feature of XGP. This sign describes the appearance of multiple rounded regions of low attenuation ( -10 to +30 Hounsfield Units) radiating outwards to the renal cortex and centred on a contracted renal pelvis (Figure 4A). These low attenuation foci are surrounded by a thin rim of higher attenuating residual renal parenchyma.

Although this appearance mimics hydronephrosis, the hypoattenuation represents infiltrating inflammation rather than calyceal distension in most cases. ${ }^{3}$

Subcortical enhancement may be demonstrated at the periphery of these multifocal hypoattenuations following intravenous contrast reflecting active inflammation. In diffuse XGP the kidney is globally enlarged with a retained reniform shape. Delayed post-contrast acquisitions usually demonstrate little contrast excretion in the affected kidney, reflecting its diminished function.

CT is the crucial imaging modality in terms of management decision-making including indications for surgery and, if indicated, via which approach. Despite the characteristic appearances on CT, significant variation of imaging features has been demonstrated in confirmed XGP cases. ${ }^{9}$ In a study of 39 cases, the majority did not have the characteristic features of maintained reniform shape and a non-functioning kidney. ${ }^{9}$ This same study reported that XGP was correctly suggested preoperatively as a possible diagnosis in only 11 patients $(28.2 \%)$. The reported incidence of obstructive nephrolithiasis in XGP is $55 \%$, and thus the presence or absence of staghorn calculus cannot be relied upon as a differentiating imaging feature, as XGP can still occur
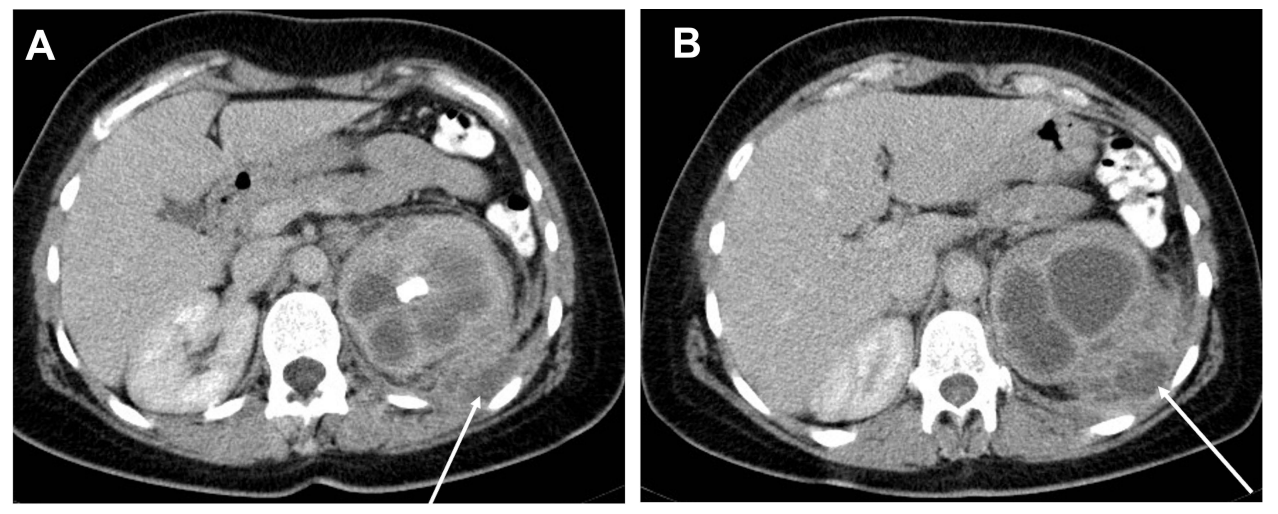

Figure 4 (A) Transaxial CT post-intravenous contrast showing multiple rounded low-attenuation foci converging on a dense calculus in a contracted renal pelvis in a "bear paw" configuration. Extrarenal extension is also evident, more conspicuous in Figure 5B (white arrow) (Elder \& Malek Stage 3 disease). (B) Extrarenal extension involving the posterior pararenal space, transversalis and deep intercostal musculature, with a small abscess cavity at the interface between transversalis and intercostal musculature (white arrow), abutting, but not eroding the rib. The posterior perirenal fascia (Zuckerkandl's) is completely engulfed. Thickening of the lateral conal and anterior perirenal fasciae and dense stranding of the properitoneal fat plane is evident (Elder \& Malek Stage 3 disease). 

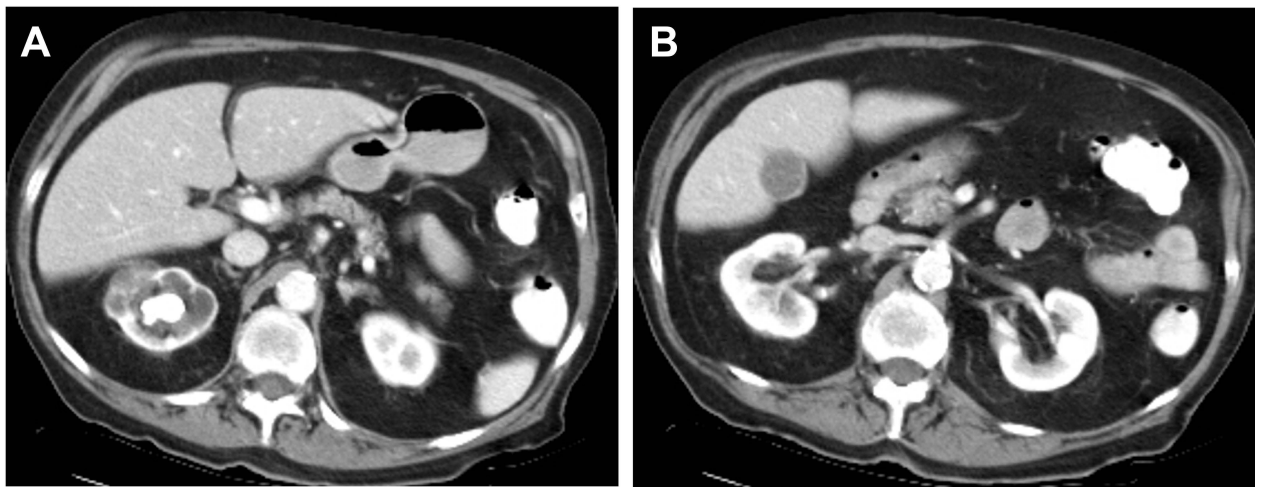

Figure 5 (A) Segmental XGP affecting the upper pole of the right kidney showing infiltrated calyces centred on a staghorn calculus in a bear-paw configuration (Elder \& Malek Stage I disease). (B) Entirely normal lower pole moiety in the same patient.

in the absence of renal calculi and stag-horn calculi are seen in the absence of XGP. The most reliable CT features in this series were found to be an enlarged kidney with bear-paw sign and extra-renal extension of the inflammatory process (Figure 4B).

Extra-renal extension including collections and fistulae are well depicted on CT, which permits demarcation of the extent and boundaries of the inflammatory process, determining if it is confined to the kidney (focal/segmental or disseminated) (Figure 5) and, in the presence of extrarenal extension, if it is confined to the perinephric or pararenal spaces and/or if it infiltrates adjacent tissues and viscera. ${ }^{10-15}$ A case series of 11 patients with XGP found that 10 patients $(91 \%)$ had extra-renal extension, three of whom $(27 \%)$ had direct extension into adjacent structures including iliopsoas muscle, abdominal wall and spleen. ${ }^{37}$

CT facilitates staging as per Malek et al as follows: ${ }^{26}$

Stage 1: Confined to renal parenchyma.

Stage 2: Renal parenchyma and peri-nephric fat.

Stage 3: Para-renal space/Retroperitoneum.

This staging was originally described in a paediatric population but is equally applicable to adults.

Complications such as infiltration of the pancreas, spleen and liver with secondary abscess formation, cutaneous and colonic fistulae as well as rib osteomyelitis are all elegantly demonstrated by CT (Figure 6). ${ }^{11-15}$

The main differential diagnosis for the $\mathrm{CT}$ appearances of XGP is primary renal malignancy; the presence of a Bear-Paw sign, a staghorn calculus and extrarenal extension of inflammation can help to differentiate XGP from primary renal malignancy. Although these are the characteristic imaging features of XGP, there is variability in their presence as described above; thus, while XGP may be the primary differential on $\mathrm{CT}$, this often requires

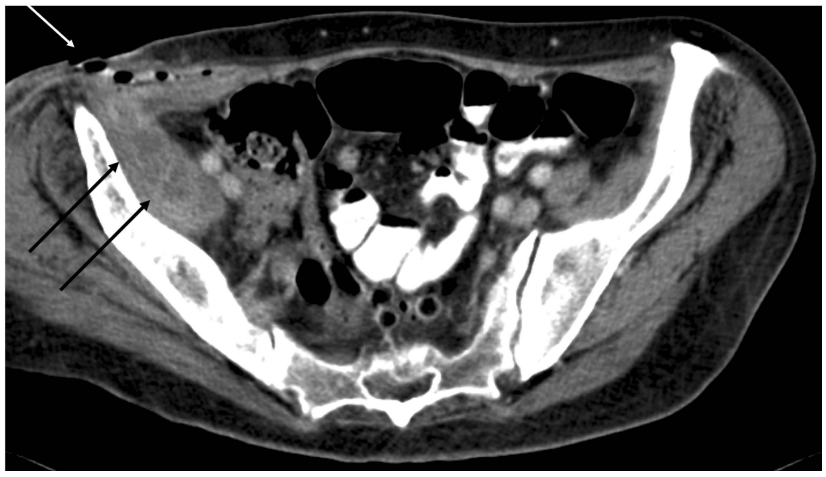

Figure 6 Multiple hypodense locules with the caudal fibres of the expanded right iliacus muscle (black arrows) representing an abscess arising from XGP in the right kidney (note the marked asymmetry with the normal left iliacus) (Elder \& Malek Stage 3 disease). Bubbles of gas are shown in the right inguinal region at the site of multiple secondary cutaneous sinuses (white arrow). Contrast is also visible within the sinuses following contrast sinography.

pathologic confirmation, given the significant overlap of findings with other conditions such as pyelonephritis, tuberculosis, malakoplakia and megalocytic interstitial nephritis. Non-radiologic investigations such as microbiology and histopathology are often required to narrow this differential to reach a specific diagnosis.

\section{Nuclear Medicine}

If DMSA is performed eg, in the paediatric population, cortical scarring will be evinced by photopenic foci. This appearance may be ambiguous initially, as photopenia may arise from either established cortical scarring or focal pyelonephritis, both of which are expected to be present. Should there be any doubt regarding the diagnosis, and a follow-up DMSA is performed, the photopenic foci will persist, confirming them as established scars rather than transient infective foci.

In diffuse XGP, DTPA and MAG-3 typically show no function in $80 \%$, or minimal residual renal function (13\%) 


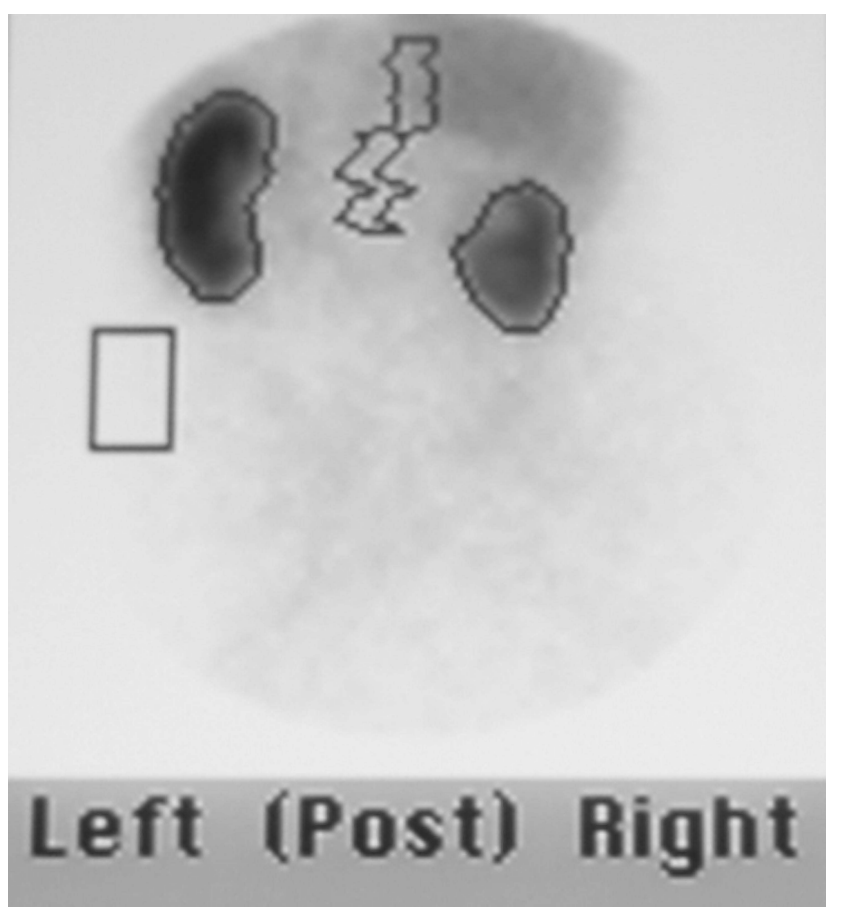

Figure 7 MAG 3 of a 64-year-old lady with XGP in the upper pole of a duplex right kidney, showing loss of function in the affected pole, but with preserved function in the lower pole moiety, the latter accounting for $38 \%$ of total renal function. Subsequent management was directed at preserving the lower pole and managing the complications relating to the upper pole. These goals were achieved using antibiotics and percutaneous interventions only.

on the affected side (Figure 7). Normal function may be observed in $7 \%{ }^{22}$ The degree of residual renal function will dictate whether renal salvage or nephron-sparing surgery is feasible, especially in the setting of focal XGP.

$\mathrm{PET} / \mathrm{CT}$ has an increasing role in the evaluation of patients with infectious or inflammatory conditions as well as in the investigation of pyrexia of unknown origin. ${ }^{38}$ Descriptions of its utility in XGP are limited to a small number of case reports. ${ }^{39,40}$

The described pattern of FDG uptake mimics that of iodinated intravenous contrast seen on CT. There is minimal uptake and relative photopenia at the sites of the hypodense collections within the affected kidney; however, intense FDG uptake at the periphery of the collections and within the overlying cortical rim has been observed, representing inflammatory hypervascularity. Low level uptake within regional lymph nodes representing benign reactive inflammation can also be seen.

Awareness of the imaging features of XGP on PET/CT is important as the condition may be encountered incidentally in oncology patients or as part of the workup for pyrexia of unknown origin. As the findings on PET/CT are non-specific (in addition to the examination's expense and limited availability, with potential overlap with malignancy), this modality does not have a primary role in the assessment of XGP. ${ }^{41,42}$

\section{MRI}

The findings on MRI are varied, presumed to reflect differing degrees of xanthoma-related inflammatory change. ${ }^{43}$ The solid component of XGP is typically isoto-hyperintense on T1-weighted imaging, representing the xanthoma cell accumulations, and isointense on T2weighted imaging compared to background renal parenchyma. MRI also demonstrates cavities and abscesses in evolution which are typically hypointense on T1weighted and hyperintense on T2-weighted imaging with possible fluid-fluid levels (Figure 8). Peri-renal inflammation is hypointense on both T1-weighted and T-2 weighted imaging representing a thick fibrinous exudate as described by Feldberg et al. ${ }^{44}$ On post-IV gadolinium dimeglumine (Gd-DTPA) imaging, rim enhancement of renal and extra-renal collections representing inflammatory hypervascularity is demonstrated; however, the material within the collections does not enhance. Enhancement of the thickened peri-renal fascia has been described, which Verswijvel et al ascribe to true extension of the xanthomatous inflammatory process. ${ }^{43}$

The appearances on MRI are non-specific and are prone to misinterpretation, given some overlap with tuberculosis and malignancy, and is as likely to cause diagnostic confusion as to secure the correct diagnosis. As a result, MRI is not routinely performed in suspected cases.

\section{Management}

Although there are a number of reports of cases managed with solely medical treatment, mainly in children, ${ }^{45}$ nephrectomy (open or laparoscopic) remains the mainstay of treatment. ${ }^{46}$ Total nephrectomy is indicated in the setting of diffuse XGP whereas nephron-sparing partial nephrectomy can be considered in focal XGP.

Nephrectomy in the setting of XGP is a challenging procedure due to the extensive perinephric inflammatory adhesions. The surgical approach will depend on the stage of disease and extent of inflammation which will dictate the need for a concurrent retroperitoneal debridement/ resection; a procedure with significant morbidity and mortality.

Earlier reports of the surgical management of XGP advocated for open nephrectomy due to the shorter operating time, less blood loss and fewer complications. ${ }^{47}$ 

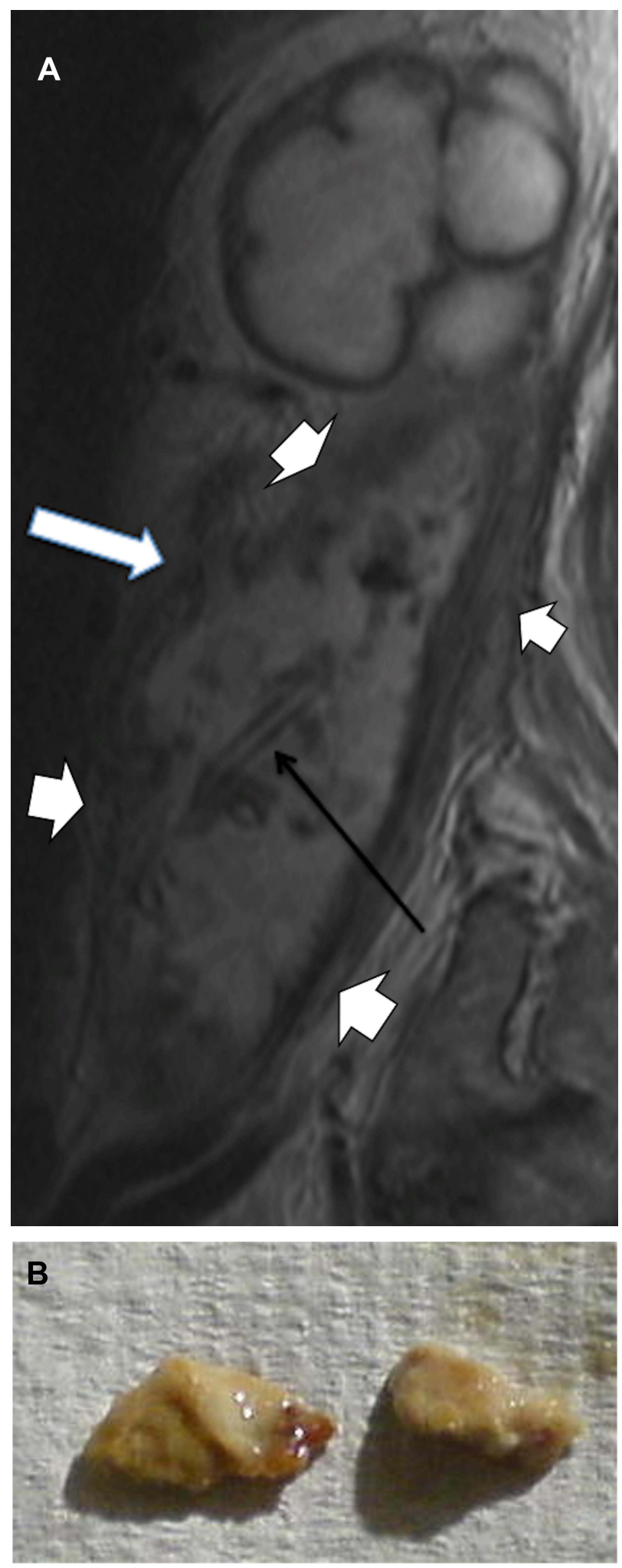

Figure 8 (A) MRI showing grossly distended calyces in the upper pole and a complex abscess cavity with a dramatically thickened, infiltrative wall (short white arrows) and innumerable foci of contained debris. The abscess arose secondary to erosion of irregular calculi through the renal pelvis, creating a sinus (long white arrow). A drainage catheter is evident within the abscess cavity (black arrow), placed via an accessed open sinus in the groin. (B) Two of the calculous fragments extracted from the abscess cavity via the groin.
Traditionally, the peri-renal and para-renal inflammation, frequently involving the renal hilum and adjacent structures, rendered the laparoscopic approach too difficult; however, advancements in skills, techniques and instruments permits the laparoscopic approach in a growing number of cases. Studies have shown favourable results for laparoscopic nephrectomy; Barboza et al in a recent case series showed less blood loss and shorter hospital stay in the laparoscopic nephrectomy group compared with open. $^{48}$

Asali et al's recent case series of 27 patients undergoing laparoscopic nephrectomy for XGP reported favourable results with only one patient requiring conversion to open procedure. ${ }^{49}$ Campanairo et al similarly showed good results with the laparoscopic approach, including in the management of Stage 2 and 3 disease. ${ }^{50}$ Unsurprisingly, a higher complication rate was associated with higher stage disease. Of the patients in their series, two were converted to open surgery, each of whom had stage 3 disease. Barboza et al compared the minimally invasive surgical (MIS) technique with open nephrectomy and demonstrated benefit in the MIS group with regard to blood loss, length of hospital stay and need for intensive care admission. In this study, $41.2 \%$ of the MIS group were completed with robotic assistance and promisingly, all of this group were completed without conversion to open. ${ }^{48}$

There are multiple case reports of patients successfully undergoing curative partial nephrectomy for focal XGP; however, it should be observed that in most of these instances the presumptive pre-operative diagnosis was RCC. ${ }^{51}$ Although there is limited data on nephronsparing surgery for XGP given its rarity, these case reports suggest it is a viable approach for focal XGP. Ongoing accumulation of evidence with this approach is anticipated, given its increasing usage.

Given that there remains limited data available on open versus laparoscopic nephrectomy for XGP, the decision regarding the optimal surgical approach will ultimately depend on local expertise and extent of inflammation demonstrated on pre-operative $\mathrm{CT}^{48}$

\section{Interventional Radiology}

Interventional radiology has a role to play in the diagnosis and management of XGP, particularly in the management of complications. As above, XGP can be difficult to differentiate from a renal malignancy, especially in its focal form. Numerous case reports describe the performance of 
percutaneous biopsy to confirm the histopathologic diagnosis of XGP and to direct subsequent management, ${ }^{52-54}$ permitting conservative management with antibiotics alone. ${ }^{55,56}$ The use of biopsy in all cases of focal XGP is advocated by some authors; however, false negative results may occur as can misdiagnosis; foam cells retrieved by needle biopsy in a patient subsequently proven to have XGP have been misinterpreted as clear cell and papillary renal cell carcinoma. ${ }^{22,57}$

Modern immunohistochemical techniques can help to avoid such misdiagnosis; XGP is positive for CD68 while Renal Cell Carcinoma is positive for CD10 and epithelial membrane antigen. Vimentin staining is unhelpful, as both $\mathrm{XGP}$ and RCC stain positive. The requirement for biopsy of XGP is still debated and varies from case to case and centre to centre. ${ }^{58}$

Diffuse XGP results in scarring and collapse of the renal collecting system with, as above, minimal to limited preservation of renal function and thus obstructive uropathy is usually not a notable feature. ${ }^{3}$ As a result, nephrostomy for the purpose of urinary diversion is only infrequently required in XGP kidneys. Leoni et al has advocated for nephrostomy placement to facilitate more precise microbiologic assessment in the setting of sterile urine cultures. ${ }^{59}$

Percutaneous aspiration of renal/extra-renal fluid collections facilitates culture and sensitivity and appropriate antibiotic management prior to more aggressive procedures. In the setting of renal/extra-renal abscess formation, pre-operative percutaneous drainage in combination with tailored antibiotic therapy allows control of the acute infectious state and improves symptoms while also resulting in a "cleaner" surgical field for subsequent nephrectomy. A case series of 27 XGP patients showed Piperacillin/Tazobactam (TZP) was the most commonly prescribed antibiotic combination ( $48.1 \%$ of patients), followed by third-generation cephalosporins $(18.5 \%))^{33}$ This approach also decreases the risk of post-surgical complications such as fistulae and impaired wound healing. ${ }^{60}$

Our group recently described a rare complication of XGP, namely extrusion of renal calculi from the renal collecting system into the retroperitoneum with psoas abscess formation in a 64 -year-old female. The extruded renal calculi were successfully retrieved by trawling the abscess cavity with an over-the-wire Fogarty balloon ultimately permitting a wholly percutaneous management, avoiding the requirement for nephrectomy. ${ }^{32}$

Upasani et al described the novel use of salvage renal artery embolisation in a 17-year-old male patient following near- complete nephrectomy, peri-renal/retroperitoneal debridement and stone retrieval which was abandoned as further dissection of the inflammatory mass was deemed unsafe. Embolisation of the arterial supply to the residual kidney was performed postoperatively with a view to preventing further proliferation of inflammation in the residual viable renal tissue. ${ }^{61}$

\section{Conclusion}

XGP is a rare aggressive granulomatous inflammatory process which can infiltrate the entirety of the kidney and surrounding tissues and organs resulting in potentially confounding symptoms, imaging features and delayed diagnosis. Nephrectomy, either open or laparoscopic, remains the definitive treatment for this inflammatory condition. Interventional Radiology techniques such as image-guided percutaneous aspiration, biopsy and drainage, stone extraction and embolisation can contribute significantly to the diagnosis and management of these patients, including salvage of abandoned surgical techniques and, rarely, preventing nephrectomy.

\section{Funding}

No funding was received for the preparation of the manuscript.

\section{Disclosure}

The authors report no conflict of interests in this work.

\section{References}

1. Schlagenhaufer F. Uber eigentumliche Staphylomykosen der Nieren und des pararenalen Bindegewebes. Frank Zeitsch Pathol. 1916;19:139-148.

2. Osterlind S. Pyelonephritis xanthomasa. Acta Chir Scand. 1944;90:369-376.

3. Craig WD, Wagner BJ, Travis MD. Pyelonephritis: radiologic-pathologic review. Radiographics. 2008;28(1):255-277. doi:10.1148/rg.281075171

4. Khalyl-Mawad J, Greco MA, Schinella RA. Ultrastructural demonstration of intracellular bacteria in xanthogranulomatous pyelonephritis. Hum Pathol. 1982;13(1):41-47. doi:10.1016/S0046-8177(82)80137-8

5. Clapton WK, Boucaut HA, Dewan PA, Bourne AJ, Byard RW. Clinicopathological features of xanthogranulomatous pyelonephritis in infancy. Pathology. 1993;25(2):110-113. doi:10.3109/ 00313029309084781

6. Antonakopoulos GN, Chapple CR, Newman J, et al. Xanthogranulomatous pyelonephritis. A reappraisal and immunohistochemical study. Arch Pathol Lab Med. 1988;112(3):275-281.

7. Youngson GG, Gray ES. Neonatal xanthogranulomatous pyelonephritis. Br J Urol. 1990;65(5):541-542. doi:10.1111/j.1464410X.1990.tb14805.x

8. Loffroy R, Guiu B, Watfa J, Michel F, Cercueil JP, Krausé D. Xanthogranulomatous pyelonephritis in adults: clinical and radiological findings in diffuse and focal forms. Clin Radiol. 2007;62 (9):884-890. doi:10.1016/j.crad.2007.04.008 
9. Zorzos I, Moutzouris V, Korakianitis G, Katsou G. Analysis of 39 cases of xanthogranulomatous pyelonephritis with emphasis on CT findings. Scand J Urol Nephrol. 2003;37(4):342-347. doi:10.1080/ 00365590310004752

10. Dawoud S, Solomon RJ, Eyerly-Webb SA, et al. Pyeloduodenal Fistula in Xanthogranulomatous Pyelonephritis: a Series of Two Cases. Perm J. 2018;22:17-150.

11. Motuzko E, Germaine P. Xanthogranulomatous pyelonephritis: the uncommon complications of the uncommon disease. Appl Radiol. 2016;45(6):32-24.

12. Numan L, Zamir H, Husainat NM, Tahboub M. Xanthogranulomatous Pyelonephritis Causing Renocolic Fistula Presenting as Symptomatic Anemia. Cureus. 2019;11(6):e4947.

13. Titus B, Gupta S, Edpao P, et al. Xanthogranulomatous Pyelonephritis With Direct Extension Into the Liver. $\mathrm{Am} \mathrm{J} \mathrm{Med.}$ 2020;133(9):1054-1055. doi:10.1016/j.amjmed.2019.12.038

14. Devrim T, Atasoy P, Tuğlu D. Xanthogranulomatous pyelonephritis: a case with rare adhesion to pancreas. CEN Case Rep. 2018;7 (1):44-47. doi:10.1007/s13730-017-0289-7

15. Eastham J, Ahlering T, Skinner E. Xanthogranulomatous pyelonephritis: clinical findings and surgical considerations. Urology. 1994;43(3):295-299. doi:10.1016/0090-4295(94)90069-8

16. Punia RS, Dhingra N, Mohan H, D’Cruz S. Amyloidosis secondary to xanthogranulomatous pyelonephritis: a rare association. Saudi J Kidney Dis Transpl. 2010;21(4):720-723.

17. Garber BB, Cendron M, Cohen R, Whitmore KE. Xanthogranulomatous pyelonephritis and amyloidosis: a rare association. J Urol. 1989;142 (1):114-116. doi:10.1016/S0022-5347(17)38678-0

18. Lauzurica R, Felip A, Serra A, et al. Xanthogranulomatous pyelonephritis and systemic amyloidosis: report of 2 new cases and the natural history of this association. J Urol. 1991;146(6):1603-1606. doi:10.1016/S0022-5347(17)38181-8

19. Li L, Parwani AV. Xanthogranulomatous pyelonephritis. Arch Pathol Lab Med. 2011;135(5):671-674. doi:10.5858/2009-0769-RSR.1

20. Upasani A, Panwar J, Chandna S, Patel D. Xanthogranulomatous pyelonephritis in early infancy. BMJ Case Rep. 2012;2012: bcr1220115305. doi:10.1136/bcr.12.2011.5305

21. Youngson GG, Gray ES. Neonatal xanthogranulomatous pyelonephritis. Br J Urol. 1990;65(5):541-542.

22. Grainger RG, Longstaff AJ, Parsons MA. Xanthogranulomatous pyelonephritis: a reappraisal. Lancet. 1982;1(8286):1398-1401. doi:10.1016/S0140-6736(82)92511-9

23. Braun G, Moussali L, Balanzar JL. Xanthogranulomatous pyelonephritis in children. J Urol. 1985;133(2):236-239. doi:10.1016/S00225347(17)48897-5

24. Levy M, Baumal R, Eddy AA. Xanthogranulomatous pyelonephritis in children. Etiology, pathogenesis, clinical and radiologic features, and management. Clin Pediatr. 1994;33(6):360-366. doi:10.1177/ 000992289403300609

25. Korkes F, Favoretto RL, Bróglio M, Silva CA, Castro MG, Perez MD. Xanthogranulomatous pyelonephritis: clinical experience with 41 cases. Urology. 2008;71(2):178-180. doi:10.1016/j.urology.2007.09.026

26. Malek RS, Elder JS. Xanthogranulomatous pyelonephritis: a critical analysis of 26 cases and of the literature. J Urol. 1978;119 (5):589-593. doi:10.1016/S0022-5347(17)57559-X

27. Goodman M, Curry T, Russell T. Xanthogranulomatous pyelonephritis (XGP): a local disease with systemic manifestations. Report of 23 patients and review of the literature. Medicine. 1979;58(2):171-181. doi:10.1097/00005792-197903000-00005

28. Ballesteros JJ, Faus R, Gironella J. Preoperative diagnosis of renal xanthogranulomatosis by serial urinary cytology: preliminary report. J Urol. 1980;124(1):9-11. doi:10.1016/S0022-5347(17)55267-2

29. Ballesteros JJ, Serrano S, Faus J, Gironella J. Urinary cytology in the diagnosis of renal xanthogranulomatosis. Eur Urol. 1983;9 (6):343-345. doi:10.1159/000474120
30. Kundu R, Baliyan A, Dhingra H, Bhalla V, Punia RS. Clinicopathological Spectrum of Xanthogranulomatous Pyelonephritis. Indian J Nephrol. 2019;29(2):111-115.

31. Hughes PM, Gupta SC, Thomas NB. Xanthogranulomatous pyelonephritis in childhood. Clin Radiol. 1990;41(5):360-362. doi:10.1016/ S0009-9260(05)81705-2

32. Hennebry J, Stroiescu A, Bolger M, Byrne C, Cross K, Ryan A. Avoidance of Nephrectomy and Percutaneous Extraction of Retroperitoneal Calculi Secondary to Xanthogranulomatous Pyelonephritis. J Vasc Interv Radiol. 2021;32(2):324-327. doi:10.1016/j.jvir.2020.09.009

33. Artiles-Medina A, Laso-García I, Lorca-álvaro J, et al. Xanthogranulomatous pyelonephritis: a focus on microbiological and antibiotic resistance profiles. BMC Urol. 2021;21(1):56. doi:10.1186/s12894-021-00800-z

34. Parsons MA, Harris SC, Longstaff AJ, Grainger RG. Xanthogranulomatous pyelonephritis: a pathological, clinical and aetiological analysis of 87 cases. Diagn Histopathol. 1983;6(3-4):203-219.

35. Goulding FJ, Moser A. Xanthogranulomatous pyelonephritis with associated renal cell carcinoma. Urology. 1984;23(4):385-386. doi:10.1016/0090-4295(84)90148-1

36. Moss BF, Potter L, Cliff A, Kumar M. Xanthogranulomatous pyelonephritis with associated renal cell carcinoma. BMJ Case Rep. 2019;12(10):e232097. doi:10.1136/bcr-2019-232097

37. Rajesh A, Jakanani G, Mayer N, Mulcahy K. Computed tomography findings in xanthogranulomatous pyelonephritis. J Clin Imaging Sci. 2011;1:45. doi:10.4103/2156-7514.84323

38. Love C, Tomas MB, Tronco GG, Palestro CJ. FDG PET of infection and inflammation. Radiographics. 2005;25(5):1357-1368. doi:10.1148/rg.255045122

39. Joshi P, Lele V, Shah H. Fluorodeoxyglucose positron emission tomography-computed tomography findings in a case of xanthogranulomatous pyelonephritis. Indian J Nucl Med. 2013;28(1):49-50. doi:10.4103/0972-3919.116809

40. Swingle CA, Baumgarten DA, Schuster DM. Xanthogranulomatous pyelonephritis characterized on PET/CT. Clin Nucl Med. 2005;30 (11):728-729. doi:10.1097/01.rlu.0000182262.16796.b3

41. Wang Z, Yan B, Wei YB, et al. Primary kidney parenchyma squamous cell carcinoma mimicking xanthogranulomatous pyelonephritis: a case report. Oncol Lett. 2016;11(3):2179-2181. doi:10.3892/ ol.2016.4200

42. Cheng G, Torigian DA, Alavi A. FDG PET/CT and MRI findings in a patient with focal xanthogranulomatous pyelonephritis mimicking cystic renal malignancy. Clin Nephrol. 2011;76(6):484-486. doi: $10.5414 / \mathrm{CN} 106762$

43. Verswijvel G, Oyen R, Van Poppel $H$, Roskams T. Xanthogranulomatous pyelonephritis: MRI findings in the diffuse and the focal type. Eur Radiol. 2000;10(4):586-589. doi:10.1007/ s003300050967

44. Feldberg MA, Driessen LP, Witkamp TD, van Leeuwen MS, van Waes PF. Xanthogranulomatous pyelonephritis: comparison of extent using computed tomography and magnetic resonance imaging in one case. Urol Radiol. 1988;10(2):92-94. doi:10.1007/BF02926545

45. Brown PS, Dodson M, Weintrub PS. Xanthogranulomatous pyelonephritis: report of nonsurgical management of a case and review of the literature. Clin Infect Dis. 1996;22(2):308-314. doi:10.1093/clinids/ 22.2.308

46. Tobias-Machado M, Lasmar MT, Batista LT, Forseto Jr PH, Juliano RV, Wroclawski ER. Laparoscopic nephrectomy in inflammatory renal disease: proposal for a staged approach. Int Braz J Urol. 2005;31(1):22-28. doi:10.1590/S1677-55382005000100005

47. Bercowsky E, Shalhav AL, Portis A, Elbahnasy AM, McDougall EM, Clayman RV. Is the laparoscopic approach justified in patients with xanthogranulomatous pyelonephritis? Urology. 1999;54(3):437-442. doi:10.1016/S0090-4295(99)00261-7 
48. Barboza MP, Nottingham CU, Calaway AC, et al. Xanthogranulomatous pyelonephritis: a comparison of open and minimally-invasive surgical approaches. J Robot Surg. 2020;1:47.

49. Asali M, Tsivian A. Laparoscopic nephrectomy in xanthogranulomatous pyelonephritis. Cent European J Urol. 2019;72(3):319-323.

50. Campanario-Pérez R, Sáiz-Marenco R, Amores-Bermúdez J, et al. Nephrectomy for the Management of Xanthogranulomatous Pyelonephritis: still a Challenging Procedure. J Endourol. 2018;32 (9):859-864. doi:10.1089/end.2018.0467

51. Mohaghegh PSM, Wong RS, Rahimi M, Shih F, Bansal R. Case report: xanthogranulomatous pyelonephritis masquerading as cystic renal cell carcinoma. Br J Biomed Sci. 2020;77(2):87-90. doi:10.1080/09674845.2019.1662977

52. Begum T, Huq ME, Ahmed M. Xanthogranulomatous pyelonephritis. BMJ Case Rep. 2016;2016:87.

53. Reul O, Waltregny D, Boverie J, de Leval J, Andrianne R. Pyélonéphrite xanthogranulomateuse pseudotumorale: diagnostic par la biopsie percutanée et succès du traitement conservateur [Pseudotumoral xanthogranulomatous pyelonephritis: diagnosis with percutaneous biopsy and success of conservative treatment]. Prog Urol. 2001;11(6):1274-1276.

54. Lizza EF, Elyaderani MK, Belis JA. Atypical presentation of xanthogranulomatous pyelonephritis: diagnosis by ultrasonography and fine needle aspiration biopsy. J Urol. 1984;132(1):95-97. doi:10.1016/ S0022-5347(17)49481-X
55. Fitouri Z, Nouira Y, Nouira K, et al. Focal xanthogranulomatous pyelonephritis: success of conservative treatment. A Case Report Tunis Med. 2008;86(10):912-915.

56. Ho CI, Wen YK, Chen ML. Xanthogranulomatous pyelonephritis successfully treated with antibiotics only. J Chin Med Assoc. 2008;71(12):643-645. doi:10.1016/S1726-4901(09)70008-5

57. Chlif M, Chakroun M, Ben Rhouma S, et al. Xanthogranulomatous pyelonephritis presenting as a pseudotumour. Can Urol Assoc J. 2016;10(1-2):E36-40. doi:10.5489/cuaj.3225

58. Ding X, Wang G, Wang T, Ma X, Wang Y. Atypical focal xanthogranulomatous pyelonephritis without clinical symptoms presenting as infiltrative renal cancer: a case report and literature review. $B M C$ Urol. 2020;20(1):63. doi:10.1186/s12894-020-00632-3

59. Leoni FA, Kinleiner P, Revol M, Zaya A, Odicio A. Píelonefritis xantogranulomatosa. Revisión de 10 casos [Xanthogranulomatous pyelonephritis: review of 10 cases]. Arch Esp Urol. 2009;62 (4):259-271. doi:10.4321/S0004-06142009000400001

60. Zafaranloo S, Gerard PS, Bryk D. Xanthogranulomatous pyelonephritis in children: analysis by diagnostic modalities. Urol Radiol. 1990;12(1):18-21. doi:10.1007/BF02923958

61. Upasani A, Barnacle A, Roebuck D, Cherian A. Combination of Surgical Drainage and Renal Artery Embolization: an Alternative Treatment for Xanthogranulomatous Pyelonephritis. Cardiovasc Intervent Radiol. 2017;40(3):470-473. doi:10.1007/s00270-016$1522-\mathrm{z}$

\section{Publish your work in this journal}

The International Journal of Nephrology and Renovascular Disease is an international, peer-reviewed open-access journal focusing on the pathophysiology of the kidney and vascular supply. Epidemiology, screening, diagnosis, and treatment interventions are covered as well as basic science, biochemical and immunological studies. The manuscript management system is completely online and includes a very quick and fair peer-review system, which is all easy to use. Visit http://www.dovepress.com/testimonials.php to read real quotes from published authors. 